\title{
A Report on the 2017 Native Language Identification Shared Task
}

\author{
Shervin Malmasi ${ }^{1,2}$, Keelan Evanini ${ }^{3}$, Aoife Cahill $^{3}$, Joel Tetreault ${ }^{4}$ \\ Robert Pugh ${ }^{5}$, Christopher Hamill ${ }^{3}$, Diane Napolitano ${ }^{3}$ and Yao Qian ${ }^{5}$ \\ ${ }^{1}$ Harvard Medical School, Boston, MA, USA \\ ${ }^{2}$ Macquarie University, Sydney, Australia \\ ${ }^{3}$ Educational Testing Service, Princeton, NJ, USA \\ ${ }^{4}$ Grammarly, New York, NY, USA \\ ${ }^{5}$ Educational Testing Service, San Francisco, CA, USA \\ shervin.malmasi@mq.edu.au, \{kevanini,acahill\}@ets.org, joel.tetreault@grammarly.com
}

\begin{abstract}
Native Language Identification (NLI) is the task of automatically identifying the native language (L1) of an individual based on their language production in a learned language. It is typically framed as a classification task where the set of L1s is known a priori. Two previous shared tasks on NLI have been organized where the aim was to identify the L1 of learners of English based on essays (2013) and spoken responses (2016) they provided during a standardized assessment of academic English proficiency. The 2017 shared task combines the inputs from the two prior tasks for the first time. There are three tracks: NLI on the essay only, NLI on the spoken response only (based on a transcription of the response and i-vector acoustic features), and NLI using both responses. We believe this makes for a more interesting shared task while building on the methods and results from the previous two shared tasks. In this paper, we report the results of the shared task. A total of 19 teams competed across the three different sub-tasks. The fusion track showed that combining the written and spoken responses provides a large boost in prediction accuracy. Multiple classifier systems (e.g. ensembles and meta-classifiers) were the most effective in all tasks, with most based on traditional classifiers (e.g. SVMs) with lexical/syntactic features.
\end{abstract}

Visit the website for more info about the task: https://sites.google.com/site/nlisharedtask/

\section{Introduction}

Native Language Identification (NLI) is the task of automatically identifying the native language (L1) of an individual based on their writing or speech in another language (L2). NLI works by identifying language use patterns that are common to certain groups of speakers that share the same native language. This process is underpinned by the presupposition that an author's linguistic background will dispose them towards particular language production patterns in their learned languages, as influenced by their mother tongue.

Predicting the native language of a writer has applications in different fields. It can be used for authorship identification (Estival et al., 2007), forensic analysis (Gibbons, 2003), tracing linguistic influence in potentially multi-author texts (Malmasi et al., 2017), and naturally to support Second Language Acquisition research (Malmasi and Dras, 2014). It can also be used in educational applications such as developing grammatical error correction systems which can personalize their feedback and model performance to the native language of the user (Rozovskaya and Roth, 2011).

Most work in NLI focused on predicting the native language of an ESL (English as a Second Language) writer based on a sample essay, although NLI has also been shown to work on other languages (Malmasi and Dras, 2015). Work by Koppel et al. (2005), Tsur and Rappoport (2007) Wong and Dras (2009), and Tetreault et al. (2012) set the stage for much of the recent research efforts. However, it was the 2013 Native Language Identification Shared Task (Tetreault et al., 2013) that led to an explosion of interest in this area by making public a large dataset developed specifically 
for this task called the TOEFL11 (Blanchard et al., 2013). In that shared task, 29 teams participated, making it one of the largest NLP competitions that year alone.

In addition to analyzing the written responses, a recent trend in NLP research has been the use of speech transcripts (generated manually or via Automatic Speech Recognition) and audio features for dialect identification (Malmasi et al., 2016), a task that involves identifying specific dialects of pluricentric languages, such as Spanish or Arabic. ${ }^{1}$ The combination of transcripts and acoustic features has also provided good results for dialect identification (Zampieri et al., 2017b), demonstrating that it is possible to improve performance by combining this information.

While there has been growing interest in using such features, the use of speech transcripts for NLI is not entirely new. In fact, the very first NLI study by Tomokiyo and Jones (2001) was based on applying a Naive Bayes classifier to transcriptions of speech from native and non-native speakers, albeit using limited data. However, this strand of NLI research has not received much attention, most likely due to the costly and laborious nature of collecting and transcribing non-native speech. Following this trend, the 2016 Computational Paralinguistics Challenge (Schuller et al., 2016) also included an NLI task based on the spoken response using the raw audio.

The NLI Shared Task 2017 attempts to combine these approaches by including a written response (essay) and a spoken response (speech transcript and i-vector acoustic features) for each candidate. The competition also allows for the fusion of all features, a novel task that has not been previously tried. Another motivation for this task was the rapid growth of deep learning methods for natural language processing tasks (Manning, 2015). In prior shared tasks, there were several barriers to using deep learning for NLP. However, deep learning has now had a positive impact on many tasks across NLP and it is an area of investigation on whether the same successes can be found in NLI.

In the following section, we provide a summary of the prior work in Native Language Identification, for both text and speech based tracks. Next, in $\S 3$, we describe the data used for training, de-

\footnotetext{
${ }^{1}$ NLI could also be framed as a dialect identification task if we assume that each L1 group has their own interlanguage/dialect which is influenced by their L1.
}

velopment, and testing in this shared task. In $\S 4$ we describe the results of each sub-task, with a short description of each team's submission. Then in $\S 5$, we discuss the commonalities and trends in and across the three sub-tasks, and present an ensemble analysis of all submissions. Finally, in $\S 6$, we offer conclusions and ideas for avenues of research in this growing field.

\section{Related Work}

NLI is most commonly framed as a supervised classification task, where features are extracted from a linguistic response produced by non-native speakers, and used to train a classification model. NLI is a recent, but rapidly growing, area of research. While some early research was conducted in the early 2000s, most work has only appeared in the last few years.

\subsection{Text-based NLI}

Most NLI research has focused on English texts where both lexical and syntactic features (often based on $n$-gram frequency profiles) have been used. Popular lexical features include character, word and lemma $n$-grams, while syntactic features are based on constituent parse trees, dependency parse features and part-of-speech tags. Support Vector Machine (SVM) models have been the most prevalent classification approach. Researchers have mainly focused on experimenting with different features and methods of combining them. While a detailed analysis of previous work is beyond the scope of this report, a comprehensive exposition of NLI research from 2001-2015, including all of the systems from the first shared task, can be found in Malmasi (2016, Section 2.3).

The winning entry for the 2013 shared task was that of Jarvis et al. (2013), achieving 83.6\% in terms of accuracy (the official metric). The features used in the system include $n$-grams of words, parts-of-speech, and lemmas. A logentropy weighting schema was used to normalize the frequencies. An L2-regularized SVM classifier was used to create a single-model system.

A notable trend in NLI has been the success of multiple classifier systems, such as ensemble classifiers (Tetreault et al., 2012). In fact, such approaches have consistently achieved state-of-theart performance on the NLI Shared Task 2013 dataset. Bykh and Meurers (2014) applied a tuned and optimized ensemble, reporting an accuracy of 
$84.82 \%$ on this data. Ionescu et al. (2014) used string kernels to perform NLI. They create several string kernels which are then combined through multiple kernel learning. They report an accuracy of $85.3 \%$ on the 2013 Test set, $1.7 \%$ higher than the winning shared task system. More recently, Malmasi and Dras (2017) presented a thorough examination of meta-classification models for NLI, achieving state-of-the-art results on three datasets from different languages, including an accuracy of $87.1 \%$ on the 2013 data.

\subsection{Speech-based NLI}

The task of speech-based NLI is closely related to the tasks of language identification and dialect identification, for which substantially more research has been conducted. For those tasks, the two main types of approach are based on acoustic features (Dehak et al., 2011) and phonotactic features (Zissman, 1996). For further details we refer the reader to Rao and Nandi (2015) and Etman and Beex (2015) which provide comprehensive overviews of the different approaches that have been taken for speech-based language and dialect identification.

The 2016 Computational Paralinguistic Challenge on NLI was designed to explore the related task of speech-based NLI in more detail. The data set for that task contained 64 hours of speech from 5,132 non-native speakers of English (approximately 45 seconds per speaker) representing the same $11 \mathrm{~L} 1$ backgrounds as the 2013 NLI Shared Task corpus. Each language was represented by recordings ranging from 458 to 485 different speakers representing a range of English speaking proficiencies. The best performing system in the challenge was that of Abad et al. (2011): their system used i-vector features that were based on Phone Log-Likelihood Ratios and achieved a performance of $81.3 \%$ (in terms of Unweighted Average Recall, which was the evaluation metric for the challenge) on the test set.

\section{Task Description and Data}

There were three tracks in the NLI Shared Task 2017: essay-only, speech-only, and fusion. The corpus consists of both written essays and orthographic transcriptions of spoken responses. These were provided by test takers in the context of a standardized assessment of a non-native speaker's ability to use and understand English for academic purposes at the university level, TOEFL ${ }^{\circledR}$ iBT. There were 11,000 test takers included in the training data (1,000 per L1) and 1,100 each for development and test (100 per L1). The 11 L1 backgrounds included in the NLI Shared Task 2017 were identical to the 2013 and 2016 shared tasks: Arabic, Chinese, French, German, Hindi, Italian, Japanese, Korean, Spanish, Telugu, and Turkish. These L1s and their language families are shown in Figure 1.

The test takers' essays and spoken responses were elicited by test questions (hereafter referred to as prompts) asking about an opinion (e.g., which of two choices the test taker would prefer) or a personal experience. A total of 8 essay prompts were included in the training and development partitions and 7 of these were represented in the test partition; a total of 9 different speaking prompts were included in the training and development partitions and 7 of these were represented in the test partition. Prompt IDs for both the essays and the spoken responses were provided with the corpus. We tried to ensure the the data was as balanced as possible by prompt (in addition to by L1), though we did not always have enough data for all L1s for some prompts.

In the essay-only track, the task was to predict the L1 of a candidate based only on an essay written in English. The essay training data consisted of the training plus development data used in the NLI Shared Task 2013, while the development essay data consisted of the test data from the 2013 task. The test data for this track was new, previously unreleased data. The average length of the essays across all three partitions was 316.2 words (SD: 77.6, Min.: 2, Max.: 796).

In the speech-only track, the task was to predict the L1 of a candidate based only on a 45second-long spoken response in English. The main source of data was a manually-created orthographic transcription of the spoken response. The average length of the speech transcriptions across all three partitions was 89.5 words (SD: 25.7, Min.: 0, Max.: 202). Unfortunately, it was not possible to distribute the raw audio for the responses. To provide a more realistic sense of the performance of a speech-based NLI system, a feature file of $i$-vectors was provided to participants who requested it. An i-vector is a fixed-length, low-dimensional representation of the sequence of frame-level acoustic measurements extracted from 


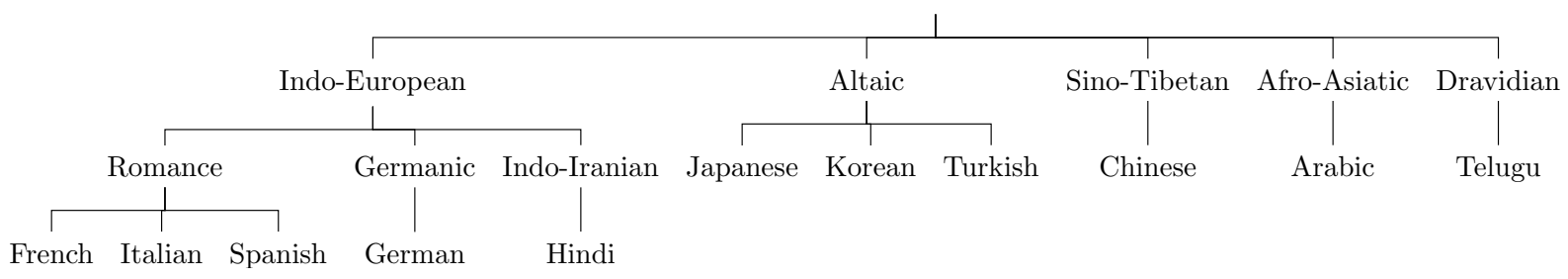

Figure 1: Language families in the task. The languages were selected to represent different families, but to also have several from within the same families. Diagram reproduced from Blanchard et al. (2013).

the speech signal (Dehak et al., 2011; Martınez et al., 2011). The dimensions of the i-vectors (800) and number of Gaussian components (1024) were tuned on the development set by using the Kaldi toolkit. ${ }^{2}$ In order to be able to distinguish the effects of new features or approaches, participants were encouraged to clearly describe the relative contribution of their features on the task both with and without the i-vector features.

In the fusion track, the task was to predict the L1 of a candidate using the combination of their written essay and spoken response.

The training and development data were released in two phases. The first phase consisted of only the essays, while the second phase consisted of the spoken transcriptions and optionally i-vectors. Simple baseline scripts that used unigram features and an SVM learner were also provided for each track.

There were both open and closed competitions for each track. In the closed competition, only the data provided could be used for training (though features based on external data sources such as language models or parsers could be included). In the open competition, additional NLI training data could be used to help improve predictions. There were no submissions to the open competition.

The test period for each track lasted 3 days, and teams could submit up to 12 systems per track. The essay-only and speech-only test phases ran concurrently. The IDs for the essay data and transcription data were generated by separate random processes for this test period. For the fusion test period, an updated package providing linked IDs between the essay and spoken transcription data was released.

\subsection{Evaluation and Ranking}

The majority of NLI research to date has reported results using accuracy as the main metric. For this task, however, we decided to use the macroaveraged F1-score as the official evaluation metric. The macro-averaged F1-score is calculated by first computing the F1-score for each class, and then taking the average across all classes (Yang and Liu, 1999). This metric favors more consistent performance across classes rather than simply measuring global performance across all samples. Accuracy was still reported for completeness.

We also used statistical significance testing for ranking purposes. McNemar's test ${ }^{3}$ (with an alpha value of 0.05 ) was applied to the ordered results to identify groups of teams where the highest and lowest results were not significantly different, and they were therefore assigned the same rank.

For comparison, we compare to two types of baselines: a random baseline and one that use a linear SVM classifier. There were three random baselines, one for each task, and five simple SVM baselines in total across the three tasks. For the essay-only task there was one baseline based on raw unigram frequencies from the essay texts. For the speech-only task there were two baselines: one an SVM based on raw unigram frequencies from the orthographic transcriptions alone, and a second SVM that combined the unigram features with the i-vectors using horizontal concatenation. For the fusion task there were two baselines: one, an SVM combining the unigrams from the essays and the transcriptions, and a second SVM combining the unigrams from the essays and the transcriptions with the i-vectors.

\footnotetext{
${ }^{2}$ http://kaldi-asr.org
}

\footnotetext{
${ }^{3}$ For more details see $\S 7.3$ of Malmasi and Dras (2017)
} 


\section{Results}

A total of 19 teams participated in the task, 17 of which submitted system description papers. Participation across the three tracks varied, with 17 participants in the essay-only track, 9 in the speech-only track, and 10 in the fusion track. The results for each track are described in the following sections. For every track we briefly outline each team's best system. Interested readers can refer to the team's paper for more details.

\subsection{Essay-only Track}

The best essay-only submission for each team, along with rankings and other details, are listed in Table 4.1. Each team's best system is briefly described below, ordered by rankings.

ItaliaNLP Lab (Cimino and Dell'Orletta, 2017) utilize a novel classifier stacking approach based on a sentence-level classifier whose predictions are used by a second document-level classifier. The sentence classifier is based on a Logistic Regression model trained on standard lexical, stylistic, and syntactic NLI features. The documentclassifier is an SVM, trained using the same features, as well as the sentence prediction labels. Their experiments indicate that inclusion of the sentence prediction features provides a small increase in performance.

CIC-FBK (Markov et al., 2017) build an SVM with multiple lexical and syntactic features. They introduce two new feature types - typed character n-grams and syntactic n-grams - and combine them with word, lemma, and POS $n$-grams, function words, and spelling error character $n$-grams. Features are weighted using log-entropy.

Groningen (Kulmizev et al., 2017) achieve their best results using a very simple system based on character 1-9 grams. Features are counted in a binary fashion and normalized via tf-idf. They also conducted experiments omitting data from some prompts during training and observe that performance can drop considerably, depending on which prompt is left out.

NRC (Goutte and Léger, 2017) explored various ways of building ensemble models to make the final prediction. Relatively simple features were used (character, word, and POS $n$-grams). Their best run for this track was a voting ensemble with 10 SVM models. tubasfs (Rama and Çöltekin, 2017) used a single SVM classifier trained on word bigrams and character 7-grams. They tried a variety of n-gram combinations and found this to work best on the development data.

UnibucKernel (Ionescu and Popescu, 2017) use different types of character-level string kernels which are combined with multiple kernel learning.

WLZ (Li and Zou, 2017) build an ensemble of single-feature SVMs fed into a multi-layer perceptron (MLP), which is a meta-classifier trained on the outputs of the base SVM classifiers. The single features are based on lexical and syntactic information and the best submission includes character, word, stem, and function word $n$-grams as well as syntactic dependencies.

Uvic-NLP (Chan et al., 2017) trained a single SVM model on word $n$-grams (1-3) and character $n$-grams (4-5). They also conducted several postevaluation experiments, improving their results to 0.8730 using an LDA meta-classifier trained on individual SVM classifiers.

ETRI-SLP (Oh et al., 2017) designed a system that was based on word $n$-gram features (with $n$ ranging from 1 to 3 ) and character $n$-gram features (with $n$ ranging from 4 to 6 ). The normalized count vectors based on these features were used to extract LSA features, which were then reduced using LDA. The count and LSA-LDA features were used to train SVM and DNN classifiers whose outputs were subsequently combined via late fusion in a DNN-based ensemble classifier.

CEMI (Ircing et al., 2017) use a Logistic Regression meta-classifier to achieve their best essay-only results. The meta-classifier is trained on the outputs of several base classifiers, which are trained on TF-IDF weighted word unigrams, word bigrams, character $n$-grams and POS $n$-grams.

RUG-SU (Bjerva et al., 2017) primarily focus on applying neural network models to NLI. Several systems are trained: A deep residual network based on word unigrams and character n-grams; a sentence-level LSTM based on POS-tagged sentences; a Logistic Regression model based on spelling error features; and a CBOW model based on document embeddings. Their best result is achieved by an ensemble combining these systems together with an SVM meta-classifier. Spelling error features did not improve overall performance. 


\begin{tabular}{|c|c|c|c|c|}
\hline Rank & Team & F1 & Acc. & Approach \\
\hline 1 & ItaliaNLP Lab & 0.8818 & 0.8818 & Stacked classifier w/ lexical and syntactic features \\
\hline 1 & CIC-FBK & 0.8808 & 0.8809 & SVM with log-entropy weighted $n$-gram and syntactic features \\
\hline 1 & Groningen & 0.8756 & 0.8755 & Linear SVM with character $n$-grams (1-9) \\
\hline 1 & NRC & 0.8740 & 0.8736 & Voting ensemble w/ SVM models using lexical/syntactic features \\
\hline 1 & tubasfs & 0.8716 & 0.8718 & SVM trained on word bigrams and char 7-grams \\
\hline 1 & UnibucKernel & 0.8695 & 0.8691 & Character-level string kernels combined w/ multiple kernel learning \\
\hline 1 & WLZ & 0.8654 & 0.8655 & MLP meta-classifier trained on SVMs w/ lexical/syntactic features \\
\hline 2 & Uvic-NLP & 0.8633 & 0.8636 & SVM trained on word and character $n$-grams \\
\hline 2 & ETRI-SLP & 0.8601 & 0.8600 & Ensemble of SVMs \& DNNs using LSA-LDA features \\
\hline 2 & CEMI & 0.8536 & 0.8536 & LogReg meta-classifier trained on word/char/POS base models \\
\hline 3 & RUG-SU & 0.8323 & 0.8318 & Ensemble of resnets, LSTM and document embeddings \\
\hline 3 & NLI-ISU & 0.8264 & 0.8264 & Logistic Regression model with word $n$-grams (1-3) \\
\hline 3 & IUCL & 0.8262 & 0.8264 & Phonetic features combined in an SVM \\
\hline 3 & GadjahMada & 0.8107 & 0.8110 & Char embeddings w/ a feed-forward NN classifier \\
\hline 4 & superliuxz & 0.7896 & 0.7900 & No paper submitted. \\
\hline 4 & ltl & 0.7676 & 0.7673 & No paper submitted. \\
\hline 5 & ut.dsp & 0.7609 & 0.7636 & $n$-gram language models over characters (3-4) and words (1-2) \\
\hline \multicolumn{2}{|c|}{ Word Unigram Baseline } & 0.7104 & 0.7109 & Linear SVM trained on word unigrams \\
\hline \multicolumn{2}{|c|}{ Random Baseline } & 0.0910 & 0.0910 & Randomly select an L1 \\
\hline
\end{tabular}

Table 1: Official results in the essay-only track. The official metric is the macro-averaged F1-score. Accuracy (Acc.) is also reported. Rankings are determined by statistical significance testing (see $§ 3.1$ ).

NLI-ISU (Vajjala and Banerjee, 2017) explored the use of $n$-grams and embeddings in their submissions. Their best run was a Logistic Regression model trained on word 1-3 grams. They also report that spell checking features, as well as word and document embeddings did not work well on the development data.

IUCL (Smiley and Kübler, 2017) investigated the use of phonetic features for the essay classification task based on the hypothesis that speakers from different L1 backgrounds may tend to use English words that match sounds in their own L1 more frequently than speakers from other L1 backgrounds. They explored three sets of phonetic features based on algorithms for fuzzy text matching (Soundex, Double Metaphone, and NYSIIS) as well as a set of features based on representations of the words using the CMU Pronouncing Dictionary. While none of these feature sets individually outperformed a system based on character $n$-grams, the addition of the Double Metaphone features to the character $n$-gram features led to a small performance improvement.
GadjahMada (Sari et al., 2017) apply a character embedding model with a feed-forward neural network classifier in the essay track. This is based on the relatively high performance of character $n$ grams in previous research. An embedding size of 25 was used with $n$-grams of length $2-5$.

ut.dsp (Mohammadi et al., 2017) utilize $n$-gram language models over words and characters. For each L1, a language model over character 3- and 4-grams as well as word unigrams and bigrams is calculated and smoothing is applied. For each text in the test set, the probably of the whole text for all language models in each class is calculated and the class with the maximum probability is chosen as the predicted label. This approach does not involve any supervised learning.

\subsection{Speech-only Track}

The best speech-only submission for each team, along with rankings and other details, are listed in Table 4.2. Each team's best system is briefly described below, ordered by rankings. 


\begin{tabular}{cllll}
\hline Rank & Team & F1 & Acc. & Approach \\
\hline 1 & UnibucKernel & 0.8755 & 0.8755 & Character-level string kernels and i-vector features \\
1 & ETRI-SLP & 0.8664 & 0.8664 & DNN ensemble with early fusion using LSA-LDA features \\
1 & CEMI & 0.8607 & 0.8609 & Ensemble of transcript \& i-vector features w/ softmax fusion \\
\hline 2 & NRC & 0.8448 & 0.8445 & Single models trained on transcript char 6-grams and i-vectors \\
2 & tubasfs & 0.8333 & 0.8336 & LDA classifier using only i-vector features \\
\hline \multicolumn{2}{c}{ Baseline: transcript + i-vector } & 0.7980 & 0.7982 & Linear SVM trained on word unigrams (transcripts) + i-vectors \\
\hline \multicolumn{2}{c}{ Baseline: transcript only } & 0.5435 & 0.5464 & Linear SVM trained on word unigrams (transcripts) \\
\hline 3 & GadjahMada & 0.5084 & 0.5073 & FFNN classifier trained on character embeddings (transcripts) \\
\hline 4 & ut.dsp & 0.4530 & 0.4536 & $n$-gram language models over transcript characters \& words \\
4 & NLI-ISU & 0.4259 & 0.4282 & Logistic Regression model w/ word $n$-grams (1-3) on transcripts \\
\hline 5 & ltl & 0.3714 & 0.3718 & No paper submitted. \\
\hline \multicolumn{2}{r}{ Random Baseline } & 0.0910 & 0.0910 & Randomly select an L1 \\
\hline
\end{tabular}

Table 2: Official results in the speech-only track. The official metric is the macro-averaged F1-score. Accuracy (Acc.) is also reported. Rankings are determined by statistical significance testing (see $\S 3.1$ ).

UnibucKernel (Ionescu and Popescu, 2017) extend their essay-only system based on characterlevel string kernels to include the transcription data, as well as an additional kernel for the i-vector features. The various models are combined using multiple kernel learning.

ETRI-SLP (Oh et al., 2017) submitted a system for the Speech task that was similar to their submission for the Essay task, although the SVM classifiers and one of the DNN classifiers were not used in the ensemble classifier. They experimented with both late fusion and early fusion for combining the text-based features with the ivectors and obtained the best results with an earlyfusion ensemble classifier.

CEMI (Ircing et al., 2017) attained their best result with an ensemble consisting of a SGD classifier trained on transcript word features and a feedforward neural network trained on the i-vector features. The final prediction is selected via softmax combination.

NRC (Goutte and Léger, 2017) use a single classifier trained on transcript character 6-grams and the i-vector features to achieve their best speech-only results.

tubasfs (Rama and Çöltekin, 2017) used an LDA classifier using only the i-vector features, a simple approach that yielded good results.
GadjahMada (Sari et al., 2017) did not use the ivector features for the speech track, applying their character embedding model from the essay track to the transcripts.

ut.dsp (Mohammadi et al., 2017) apply their $n$ gram language model from the essay-only track to the transcripts.

NLI-ISU (Vajjala and Banerjee, 2017) did not use the i-vector features for the speech track, instead applying their $n$-gram based model from the essay track. They report that the essay features do not work very well for transcripts, hypothesizing that this may be due to the shorter texts.

\subsection{Fusion Track}

The best fusion submission for each team, along with rankings and other details, are listed in Table 4.3. Each team's best system is briefly described below, ordered by rankings.

UnibucKernel (Ionescu and Popescu, 2017) extend their speech system to also include essays, in addition to the transcripts and i-vectors. The models are combined via multiple kernel learning.

CEMI (Ircing et al., 2017) obtain their best results using a neural network based meta-classifier. They use several isolated feed-forward neural network models, each trained on one feature type. Features include word, character, and POS $n$ grams (from transcripts/essays) plus i-vectors. The outputs from the networks are fused using softmax combination to predict the final label. 


\begin{tabular}{cllcl}
\hline Rank & Team & F1 & Acc. & Approach \\
\hline 1 & UnibucKernel & 0.9319 & 0.9318 & Character-level string kernels and i-vector features \\
1 & CEMI & 0.9257 & 0.9255 & NN meta-classifier over lexical/syntactic/i-vector features \\
1 & ETRI-SLP & 0.9220 & 0.9218 & DNN ensemble with early fusion using LSA-LDA features \\
1 & NRC & 0.9193 & 0.9191 & Voting ensemble w/ half sampling to choose the SVM models \\
\hline 2 & tubasfs & 0.9175 & 0.9173 & Ensemble w/ word/char $n$-grams (essay/transcript) \& i-vectors \\
\hline 3 & GadjahMada & 0.8414 & 0.8409 & FFNN trained on essay character embeddings and i-vectors \\
3 & L2F & 0.8377 & 0.8391 & BPE $n$-grams, NN fusion, i-vector post-processing \\
3 & ZCD & 0.8358 & 0.8355 & Ensemble of word/char. $n$-gram and i-vector SVM classifiers \\
\hline Baseline: essay/transcript/i-vector & 0.7901 & 0.7909 & SVM trained on word unigrams (essay/transcript) + i-vectors \\
\hline \multicolumn{2}{c}{ Baseline: Essay + Transcript } & 0.7786 & 0.7791 & Linear SVM trained on word unigrams (essays + transcripts) \\
\hline 4 & ut.dsp & 0.7748 & 0.7764 & $n$-gram language models over chars/words (essay+transcript) \\
\hline 5 & ltl & 0.7346 & 0.7345 & No paper submitted. \\
\hline & Random Baseline & 0.0910 & 0.0910 & Randomly select an L1 \\
\hline
\end{tabular}

Table 3: Official results in the fusion track. The official metric is the macro-averaged F1-score. Accuracy (Acc.) is also reported. Team rankings are determined by statistical significance testing (see $\S 3.1$ ).

ETRI-SLP (Oh et al., 2017) submitted a system for the Fusion task that was similar to their submissions for the Essay and Speech tasks, although the SVM and DNN classifiers were not used in the ensemble classifier; their ensemble classifier for the fusion task only combined the LSA-LDA features and the i-vectors. As with the Speech task, they experimented with both late fusion and early fusion for combining the text-based features with the i-vectors and obtained the best results with an early-fusion ensemble classifier.

NRC (Goutte and Léger, 2017) explored various ways of building ensemble models to make the final prediction. Relatively simple features were used (character, word, and POS $n$-grams). For the fusion track, their best submission used half sampling which uses one half of the data to estimate the best number of models to include in the final voting ensemble, and the other half to estimate which models to include.

tubasfs (Rama and Çöltekin, 2017) obtain their best result with an ensemble model based on mean probability combination. The ensemble includes individual SVM models trained on word and character $n$-grams from essays and transcripts, and an LDA classifier trained on the i-vector features.

GadjahMada (Sari et al., 2017) extended their essay-based character embedding model to include i-vectors for the fusion track. They did not use the speech transcript data.
L2F (Kepler et al., 2017) designed a system that combined three types of text-based classifiers (an RNN with a bidirectional GRU layer, a Naive Bayes classifier with byte $n$-grams, and a Naive Bayes classifier with $n$-grams based on representations of the words using Byte Pair Encoding) with versions of the i-vector features that were postprocessed using centering and whitening in an attempt to reduce channel variability. These classifiers were combined together in a Neural Network fusion approach and the authors demonstrated that the i-vector features were the main driver of performance.

ZCD (Zampieri et al., 2017a) used an approach based on ensembles of multiple SVM classifiers. Separate SVM classifiers were trained using character $n$-grams (with $n$ ranging from 1 to 10 ) and word $n$-grams (with $n$ ranging from 1 to 2 ). Individual classifiers with cross-validation performance lower than 0.8 were retained in the ensemble; the classifiers that were retained were based on character $n$-grams with $n$ in $6,7,8$. These $n$ gram-based classifiers were then combined into an ensemble with a classifier based on the i-vector features and the majority vote from the ensemble was taken as the final prediction.

ut.dsp (Mohammadi et al., 2017) apply their $n$ gram language model from the essay-only track to the combination of essays and transcripts. 


\section{Discussion and Analysis}

In this section we synthesize the overarching findings from this edition of the NLI shared task.

\subsection{Primary Trends}

Multiple Classifier Systems are very effective. Almost all of the top ranked teams employed some type of multiple classifier system, including metaclassifiers (classifier stacking), ensemble combination methods (voting and probability based fusion), and multiple kernel learning. Their use has become much more prevalent compared to the previous shared task.

Lexical n-grams are the best single feature type. Surface form features such as word and character $n$-grams continue to be the powerhouse feature for the text classification tasks. Evidence from various participants suggests that high-order character $n$-grams (as high as $n=10$ ) are extremely useful for this task. This is likely because when extracted across word boundaries, these features capture not only sub-word (e.g. morphological) information, but also dependencies between words. However, it should also be noted that the top systems in all tracks made use of syntactic features which can give them a slight performance boost. This is not surprising as it has been shown that lexical and syntactic features each capture diverse types of information that are complementary (Malmasi and Cahill, 2015).

Feature weighting schemes are important. Similar to past results, many of the top teams apply a form of feature weighting (such as TF-IDF or log-entropy) to their data.

Acoustic features are highly informative for speech-based NLI. Using only text-based features over the transcripts did not work well, and teams that did not utilize the i-vector features performed much worse in the speech-only track. The top-ranked teams combined the transcripts and ivectors.

Speech transcript features did not perform well. Teams that used only the transcript features did not fare well in the speech track. This could be due to the different types of linguistic phenomena that are present in spontaneous speech, which may be less informative than those found in the essays.
Various teams also hypothesize that this may potentially be due to their relatively shorter lengths compared to the essays (see $\S 3$ for stats).

Fusion of writing and speech features provides the best results. The substantial performance increase between the essay/speech tracks and the fusion track indicates that the acoustic features are complementary and lead to much more reliable results.

Traditional classifier models continue to dominate text classification tasks. It has been noted that traditional supervised learning models outperform newer deep learning approaches on highdimensional text classification tasks (Malmasi et al., 2016, §6.2). The results from this NLI task do not provide any evidence to suggest otherwise; almost all of the top teams in the essay-only track used an SVM or similar linear model. Uvic-NLP (Chan et al., 2017) compared SVMs and neural network models, finding that SVM models achieve better results with shorter training times.

Average performance is much higher than 2013. Although much of the training data remains the same, the submissions were much more competitive than the first NLI shared tasks. This is likely due to NLI being a much more established task, as well as the aforementioned prevalence of more sophisticated models such as metaclassifiers.

A number of open questions remain. For example, it is not clear if any one approach is dominant across all tracks as most of the top-ranked teams in the essay track did not participate in the other tracks. It is hard to say how well their systems would have done in the other tracks, but the trends from the teams who did participate in all tracks suggest that their approaches could have done well.

It is also clear that ensemble-based systems attain some of the best results, but while we note that meta-classifiers were particularly popular, it is difficult to draw conclusions about the best approach as most teams used different configurations (e.g. different base classifiers and meta-classifier models). A comprehensive and detailed study is needed to provide an empirical comparison of the different methods. 


\begin{tabular}{r|c|ccc}
\hline & $\mathbf{2 0 1 3}$ & \multicolumn{3}{|c}{$\mathbf{2 0 1 7}$} \\
& & Essay & Speech & Fusion \\
\hline \# Systems & 29 & 17 & 9 & 10 \\
\hline Shared Task Best & 0.8359 & 0.8818 & 0.8755 & 0.9319 \\
\hline Oracle & 0.9791 & 0.9628 & 0.9572 & 0.9809 \\
Accuracy@3 & 0.9555 & 0.9592 & 0.9508 & 0.9764 \\
Accuracy@ @ & 0.9218 & 0.9501 & 0.9290 & 0.9700 \\
Plurality Vote & 0.8425 & 0.8793 & 0.8508 & 0.9319 \\
\hline
\end{tabular}

Table 4: Oracle results on the NLI 2013 and 2017 shared task systems. The ensemble includes each team's best system in each track. Results are reported as the macro-averaged F1-score.

\subsection{Ensemble Analysis}

One interesting research question is to measure the upper-bound on accuracy for this year's task. This can be measured by treating each team's best submission as an independent system, and combining the results using ensemble methods such as a plurality vote or an oracle. This type of analysis has previously been applied to the NLI 2013 task and shown to be helpful in other work (Malmasi et al., 2015). Following the approach of Malmasi et al. (2015), we apply the following combination methods to the 2017 data.

Plurality Voting: This is the standard combination strategy that selects the label with the highest number of votes, regardless of the overall percentage of votes it received (Polikar, 2006). This differs from a majority vote combiner where a label must obtain over $50 \%$ of the votes.

Oracle: An oracle is a type of fusion method that assigns the correct class label for an instance if any of the classifiers in the ensemble produces the correct label for that data point. This method has previously been used to analyze the limits of majority vote classifier combination (Kuncheva et al., 2001). It can help quantify the potential upper limit of an ensemble's performance on the given data and how this performance varies with different ensemble configurations and combinations.

Accuracy@ N: To account for the possibility that a classifier may randomly predict the correct label (with a probability determined by the random baseline) and thus exaggerate the oracle score, an Accuracy@ $N$ combiner has been proposed (Malmasi et al., 2015). This method is inspired by the "Precision at $k$ " metric from Information Retrieval (Manning et al., 2008) which measures precision at fixed low levels of results (e.g. the top 10 re- sults). Here, it is an extension of the Plurality vote combiner where instead of selecting the label with the highest votes, the labels are ranked by their vote counts and a sample is correctly classified if the true label is in the top $N$ ranked candidates. ${ }^{4}$ Another way to view it is as a more restricted version of the Oracle combiner that is limited to the top $N$ ranked candidates in order to minimize the influence of a single classifier having chosen the correct label by chance. In this study we experiment with $N=2$ and 3 . We also note that setting $N=1$ is the same as the Plurality voting method.

We applied the above combiners to all three tracks in the NLI 2017 task. The results are presented in Table 4. The results for each track are compared against the best system in the shared task. The equivalent results from the NLI 2013 shared task are also included for comparison.

We note that the 2017 oracle performance is similar to that of 2013, despite having fewer systems. The Accuracy@2 results are also substantially higher. Another difference in 2017 is that the voting ensemble did not outperform the single best system in any track, which was the case in 2013. Taken together, these trends seem to suggest that the 2017 entries were more accurate, rather than the test set being easier to classify (in which case we would have expected higher oracle results).

Results from the Accuracy@2 combiner show that a great majority of the texts are close to being correctly classified: this value is significantly higher than the plurality combiner and not much lower than the oracle itself. This shows that the correct label receives a significant portion of the votes, and when not the winning label, it is often the runner-up.

\footnotetext{
${ }^{4}$ In case of ties we choose randomly from the labels with the highest number of votes.
} 


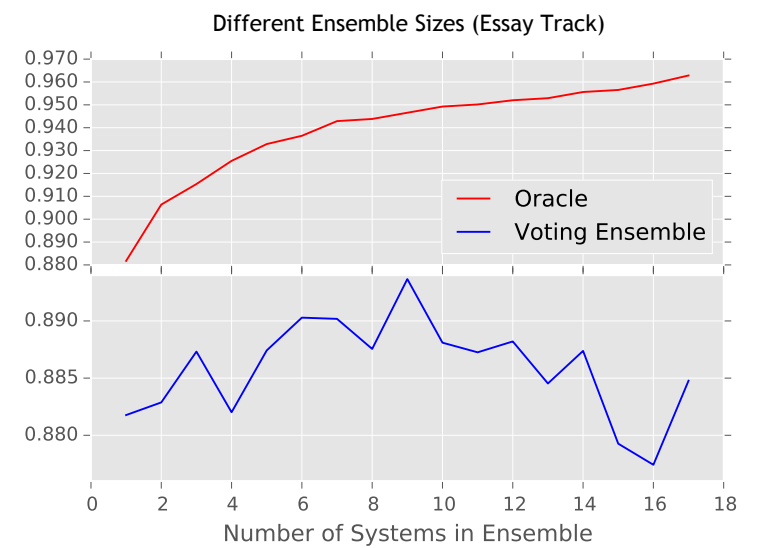

Figure 2: Results (macro-F1) for ensembles of different sizes using each team's best system in the Essay track. Systems are added according to their absolute rank. Oracle combination (top) and plurality voting (bottom) are shown.

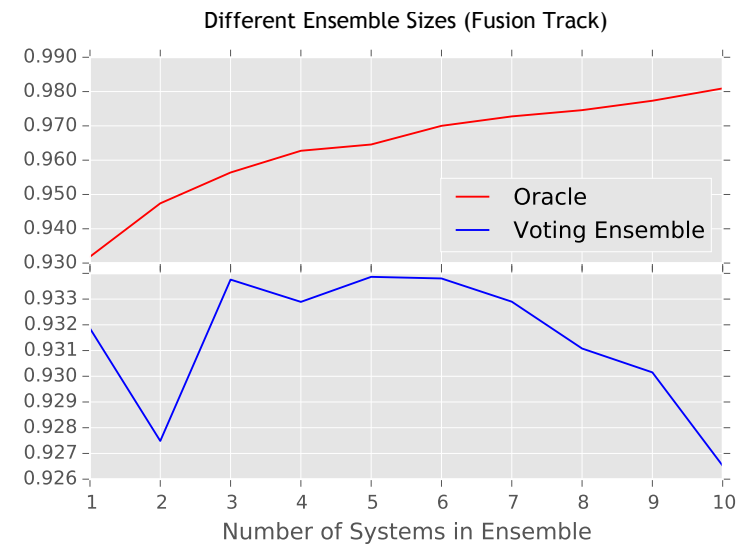

Figure 3: Results (macro-F1) for creating ensembles of different sizes using each team's best submission in the Fusion track. Systems are added according to their absolute rank. Oracle combination (top) and plurality voting (bottom) are shown.

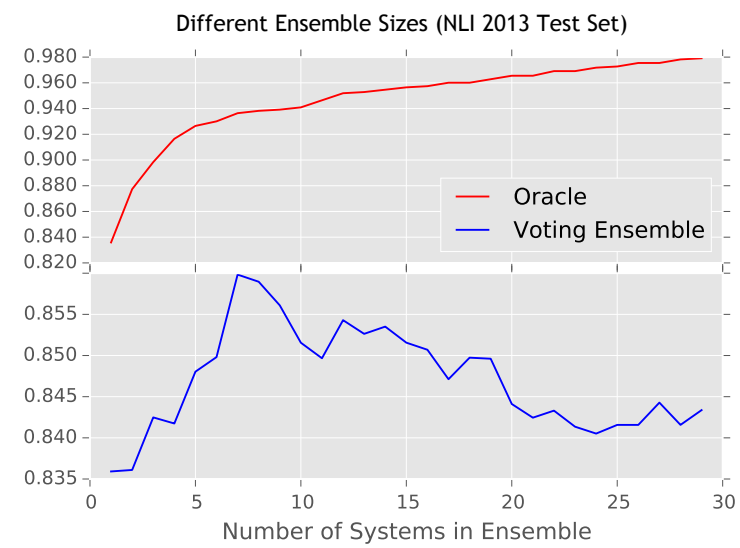

Figure 4: Results (macro-F1) for ensembles of different sizes using each team's best system in the NLI Shared Task 2013 (test set). Systems are added in order of their rank. Oracle combination (top) and plurality voting (bottom) are shown.
It is also evident that the results for the fusion track are much higher, again highlighting the utility of combining multiple modalities for NLI.

In addition to using each team's best system, We also experimented with creating ensembles of different sizes. For each track we created $N$ ensembles $E_{1} \ldots E_{N}$, with $N$ being the number of systems in that track. Each ensemble $E_{n}$ contains the top $n$ systems in the given track, so that the first ensemble contains only the top system, the second contains the top two systems, and so on, with the final ensemble containing every team's system.

This analysis enables us to assess the ensemble performance as more predictions are added. The results for the Oracle and Plurality Vote ensembles in the essay and fusion tracks are shown in Figure 2 and Figure 3. For comparison we also include the ensemble combinations generated from the 2013 test set, as shown in Figure 4.

For both tracks we observe that oracle accuracy increases as more systems are added, which is to be expected. For voting combination, performance increases as the top systems are added, but then begins to drop off as errors are introduced from the less accurate systems. This suggests that it might be possible to develop a system that performs slightly better than the top-ranked system.

On balance, the analysis presented in this section suggests that it will be challenging to develop NLI systems that attain statistically significant gains on this data.

\section{Conclusion and Future Work}

We presented the results of the NLI Shared Task 2017. This edition of the task introduced the use of transcriptions and i-vector features for speechbased NLI, as well the as the fusion task which jointly uses the spoken and written responses.

The task attracted strong participation with 19 entrants, many of whom developed systems that built on recent research in the field. The fusion track demonstrated that the combination of the written and spoken response can provide a substantial boost in classification accuracy. Multiple classifier systems (such as ensembles and meta-classifiers) were the most effective across all tracks. Mainly using lexical and syntactic features, models were mostly based on traditional classification methods (e.g. SVMs) which were not outperformed by deep learning approaches. Taken together, their results have generated a number of 
new insights for this task, and serve as a building block for future work. The results obtained here will also provide an important benchmark for assessing future results.

There are a number of avenues for future NLI research. Although we were not able to include the raw audio data in this task, its inclusion in the speech and fusion tasks could be an interesting addition. The expansion of the L1 classes to include a larger number of linguistically diverse languages can also be insightful. Most NLI research to date has been limited to approximately a dozen languages, so it is not clear how these systems will fare as the number of classes increases.

The relatively low performance of transcriptionbased features also merits further investigation. A first step would be to assess whether the primary issue is related to the shorter lengths of the texts. This hypothesis can be tested by obtaining transcripts of longer spoken responses, or even artificially creating longer texts by concatenating the existing data.

Finally, the essay-based NLI results obtained on English L2 data have been replicated on a range of other languages (Malmasi and Dras, 2015). It would be interesting to see to what degree the speech-based NLI methodologies would work on other languages. The paucity of spoken responses from learners of languages other than English makes this a challenging research question.

\section{References}

Alberto Abad, Eugénio Ribeiro, Fábio Kepler, Ramon Astudillo, and Isabel Trancoso. 2011. Exploiting phone log-likelihood ratio features for the detection of the native language of non-native english speakers. In Proceedings of Interspeech. San Francisco, USA, pages 2413-2417.

Johannes Bjerva, Gintarè Grigonytė, Robert Östling, and Barbara Plank. 2017. Neural Networks and Spelling Features for Native Language Identification. In Proceedings of the 12th Workshop on Building Educational Applications Using NLP. Association for Computational Linguistics, Copenhagen, Denmark.

Daniel Blanchard, Joel Tetreault, Derrick Higgins, Aoife Cahill, and Martin Chodorow. 2013. TOEFL11: A Corpus of Non-Native English. Technical report, Educational Testing Service.
Serhiy Bykh and Detmar Meurers. 2014. Exploring Syntactic Features for Native Language Identification: A Variationist Perspective on Feature Encoding and Ensemble Optimization. In Proceedings of COLING 2014, the 25th International Conference on Computational Linguistics: Technical Papers. Dublin, Ireland, pages 1962-1973.

Sophia Chan, Maryam Honari Jahromi, Benjamin Benetti, Aazim Lakhani, and Alona Fyshe. 2017. Ensemble Methods for Native Language Identification. In Proceedings of the 12th Workshop on Building Educational Applications Using NLP. Association for Computational Linguistics, Copenhagen, Denmark.

Andrea Cimino and Felice Dell'Orletta. 2017. Stacked Sentence-Document Classifier Approach for Improving Native Language Identification. In Proceedings of the 12th Workshop on Building Educational Applications Using NLP. Association for Computational Linguistics, Copenhagen, Denmark.

Najim Dehak, Pedro A Torres-Carrasquillo, Douglas Reynolds, and Reda Dehak. 2011. Language recognition via i-vectors and dimensionality reduction. In Twelfth Annual Conference of the International Speech Communication Association.

Dominique Estival, Tanja Gaustad, Son Bao Pham, Will Radford, and Ben Hutchinson. 2007. Author profiling for English emails. In Proceedings of the 10th Conference of the Pacific Association for Computational Linguistics. Melbourne, Australia, pages 263-272.

Asmaa Etman and A. A. Louis Beex. 2015. Language and Dialect Identification: A survey. In SAI Intelligent Systems Conference. London, UK, pages 220231.

J. Gibbons. 2003. Forensic Linguistics: An Introduction to Language in the Justice System..

Cyril Goutte and Serge Léger. 2017. Exploring Optimal Voting in Native Language Identification. In Proceedings of the 12th Workshop on Building Educational Applications Using NLP. Association for Computational Linguistics, Copenhagen, Denmark.

Radu Tudor Ionescu and Marius Popescu. 2017. Can string kernels pass the test of time in Native Language Identification? In Proceedings of the 12th Workshop on Building Educational Applications Using NLP. Association for Computational Linguistics, Copenhagen, Denmark.

Radu Tudor Ionescu, Marius Popescu, and Aoife Cahill. 2014. Can characters reveal your native language? A language-independent approach to native language identification. In Proceedings of the 2014 Conference on Empirical Methods in Natural Language Processing (EMNLP). Association for Computational Linguistics. 
Pavel Ircing, Jan Švec, Zbyněk Zajíc, Barbora Hladká, and Martin Holub. 2017. Combining Textual and Speech Features in the NLI Task Using State-ofthe-Art Machine Learning Techniques. In Proceedings of the 12th Workshop on Building Educational Applications Using NLP. Association for Computational Linguistics, Copenhagen, Denmark.

Scott Jarvis, Yves Bestgen, and Steve Pepper. 2013. Maximizing Classification Accuracy in Native Language Identification. In Proceedings of the Eighth Workshop on Innovative Use of NLP for Building Educational Applications. Association for Computational Linguistics, Atlanta, Georgia, pages 111118.

Fabio N. Kepler, Ramon F. Astudillo, and Alberto Abad. 2017. Fusion of Simple Models for Native Language Identification. In Proceedings of the 12th Workshop on Building Educational Applications Using NLP. Association for Computational Linguistics, Copenhagen, Denmark.

Moshe Koppel, Jonathan Schler, and Kfir Zigdon. 2005. Automatically determining an anonymous author's native language. Intelligence and Security Informatics pages 41-76.

Artur Kulmizev, Bo Blankers, Johannes Bjerva, Malvina Nissim, Gertjan van Noord, Barbara Plank, and Martijn Wieling. 2017. The Power of Character Ngrams in Native Language Identification. In Proceedings of the 12th Workshop on Building Educational Applications Using NLP. Association for Computational Linguistics, Copenhagen, Denmark.

Ludmila I Kuncheva, James C Bezdek, and Robert PW Duin. 2001. Decision templates for multiple classifier fusion: an experimental comparison. Pattern Recognition 34(2):299-314.

Wen Li and Liang Zou. 2017. Classifier Stacking for Native Language Identification. In Proceedings of the 12th Workshop on Building Educational Applications Using NLP. Association for Computational Linguistics, Copenhagen, Denmark.

Shervin Malmasi. 2016. Native Language Identification: Explorations and Applications. Ph.D. thesis. http://hdl.handle.net/1959.14/1110919.

Shervin Malmasi and Aoife Cahill. 2015. Measuring Feature Diversity in Native Language Identification. In Proceedings of the Tenth Workshop on Innovative Use of NLP for Building Educational Applications. Association for Computational Linguistics, Denver, Colorado, pages 49-55. http://aclweb.org/anthology/W15-0606.

Shervin Malmasi and Mark Dras. 2014. Language Transfer Hypotheses with Linear SVM Weights. In Proceedings of the 2014 Conference on Empirical Methods in Natural Language Processing (EMNLP). Association for Computational Linguistics, Doha, Qatar, pages 1385-1390. http://aclweb.org/anthology/D14-1144.
Shervin Malmasi and Mark Dras. 2015. Multilingual Native Language Identification. In Natural Language Engineering.

Shervin Malmasi and Mark Dras. 2017. Native Language Identification using Stacked Generalization. arXiv preprint arXiv:1703.06541 .

Shervin Malmasi, Mark Dras, Mark Johnson, Lan Du, and Magdalena Wolska. 2017. Unsupervised Text Segmentation Based on Native Language Characteristics. In Proceedings of the 55th Annual Meeting of the Association for Computational Linguistics (ACL 2017). Vancouver, Canada.

Shervin Malmasi, Joel Tetreault, and Mark Dras. 2015. Oracle and Human Baselines for Native Language Identification. In Proceedings of the 10th BEA workshop. pages 172-178.

Shervin Malmasi, Marcos Zampieri, Nikola Ljubešić, Preslav Nakov, Ahmed Ali, and Jörg Tiedemann. 2016. Discriminating between Similar Languages and Arabic Dialect Identification: A Report on the Third DSL Shared Task. In Proceedings of the VarDial Workshop. Osaka, Japan.

Christopher D Manning. 2015. Computational linguistics and deep learning. Computational Linguistics 41(4):701-707.

Christopher D Manning, Prabhakar Raghavan, and Hinrich Schütze. 2008. Evaluation in information retrieval. In Introduction to Information Retrieval, Cambridge university press Cambridge, pages 151175.

Ilia Markov, Lingzhen Chen, Carlo Strapparava, and Grigori Sidorov. 2017. CIC-FBK Approach to Native Language Identification. In Proceedings of the 12th Workshop on Building Educational Applications Using NLP. Association for Computational Linguistics, Copenhagen, Denmark.

David Martınez, Oldrich Plchot, Lukás Burget, Ondrej Glembek, and Pavel Matejka. 2011. Language recognition in ivectors space. In Proceedings of Interspeech. Firenze, Italy, pages 861-864.

Elham Mohammadi, Hadi Veisi, and Hessam Amini. 2017. Native Language Identification Using a Mixture of Character and Word N-grams. In Proceedings of the 12th Workshop on Building Educational Applications Using NLP. Association for Computational Linguistics, Copenhagen, Denmark.

Yoo Rhee Oh, Hyung-Bae Jeon, Hwa Jeon Song, YunKyung Lee, Jeon-Gue Park, and Yun-Keun Lee. 2017. A deep-learning based native-language classification by using a latent semantic analysis for the NLI Shared Task 2017. In Proceedings of the 12th Workshop on Building Educational Applications Using NLP. Association for Computational Linguistics, Copenhagen, Denmark. 
Robi Polikar. 2006. Ensemble based systems in decision making. Circuits and systems magazine, IEEE 6(3):21-45.

Taraka Rama and Çağrı Çöltekin. 2017. Fewer features perform well at Native Language Identification task. In Proceedings of the 12th Workshop on Building Educational Applications Using NLP. Association for Computational Linguistics, Copenhagen, Denmark.

K. Sreenivasa Rao and Dipanjan Nandi. 2015. Language identificationa brief review. In Wim Kouwenhoven, editor, Language Identification Using Excitation Source Features, Springe, chapter 2, pages 1130 .

Alla Rozovskaya and Dan Roth. 2011. Algorithm selection and model adaptation for esl correction tasks. In Proceedings of the 49th Annual Meeting of the Association for Computational Linguistics: Human Language Technologies. Association for Computational Linguistics, Portland, Oregon, USA, pages 924-933. http://www.aclweb.org/anthology/P111093.

Yunita Sari, Muhammad Rifqi Fatchurrahman, and Meisyarah Dwiastuti. 2017. A Shallow Neural Network for Native Language Identification with Character N-grams. In Proceedings of the 12th Workshop on Building Educational Applications Using NLP. Association for Computational Linguistics, Copenhagen, Denmark.

Björn Schuller, Stefan Steidl, Anton Batliner, Julia Hirschberg, Judee K. Burgoon, Alice Baird, Aaron Elkins, Yue Zhang, Eduardo Coutinho, and Keelan Evanini. 2016. The INTERSPEECH 2016 Computational Paralinguistics Challenge: Deception, Sincerity \& Native Language. In Interspeech 2016. pages 2001-2005. https://doi.org/10.21437/Interspeech.2016-129.

Charese Smiley and Sandra Kübler. 2017. Native Language Identification using Phonetic Algorithms. In Proceedings of the 12th Workshop on Building Educational Applications Using NLP. Association for Computational Linguistics, Copenhagen, Denmark.

Joel Tetreault, Daniel Blanchard, and Aoife Cahill. 2013. A report on the first native language identification shared task. In Proceedings of the Eighth Workshop on Innovative Use of NLP for Building Educational Applications. Association for Computational Linguistics, Atlanta, GA, USA.

Joel Tetreault, Daniel Blanchard, Aoife Cahill, and Martin Chodorow. 2012. Native tongues, lost and found: Resources and empirical evaluations in native language identification. In Proceedings of COLING 2012. The COLING 2012 Organizing Committee, Mumbai, India, pages 2585-2602. http://www.aclweb.org/anthology/C12-1158.
Laura Mayfield Tomokiyo and Rosie Jones. 2001. You're not from 'Round here, are you?: Naive Bayes detection of Non-native utterance text. In Proceedings of the second meeting of the North American Chapter of the ACL (NAACL). Association for Computational Linguistics, Pittsburgh, PA.

Oren Tsur and Ari Rappoport. 2007. Using Classifier Features for Studying the Effect of Native Language on the Choice of Written Second Language Words. In Proceedings of the Workshop on Cognitive Aspects of Computational Language Acquisition. Association for Computational Linguistics, Prague, Czech Republic, pages 9-16. http://www.aclweb.org/anthology/W/W07/W070602.

Sowmya Vajjala and Sagnik Banerjee. 2017. A study of N-gram and Embedding Representations for $\mathrm{Na}$ tive Language Identification. In Proceedings of the 12th Workshop on Building Educational Applications Using NLP. Association for Computational Linguistics, Copenhagen, Denmark.

Sze-Meng Jojo Wong and Mark Dras. 2009. Contrastive Analysis and Native Language Identification. In Proceedings of the Australasian Language Technology Association Workshop 2009. Sydney, Australia, pages 53-61. http://www.aclweb.org/anthology/U09-1008.

Yiming Yang and Xin Liu. 1999. A re-examination of text categorization methods. In Proceedings of the 22nd annual international ACM SIGIR conference on Research and development in information retrieval. ACM, pages 42-49.

Marcos Zampieri, Alina Maria Ciobanu, and Liviu P. Dinu. 2017a. Native Language Identification on Text and Speech. In Proceedings of the 12th Workshop on Building Educational Applications Using NLP. Association for Computational Linguistics, Copenhagen, Denmark.

Marcos Zampieri, Shervin Malmasi, Nikola Ljubešić, Preslav Nakov, Ahmed Ali, Jörg Tiedemann, Yves Scherrer, and Noëmi Aepli. 2017b. Findings of the VarDial Evaluation Campaign 2017. In Proceedings of the Fourth Workshop on NLP for Similar Languages, Varieties and Dialects (VarDial). Valencia, Spain, pages 1-15.

Marc Zissman. 1996. Comparison of four approaches to automatic language identification of telephone speech. IEEE Transactions on Speech and Audio Processing 4(1):31-44. 